\title{
From candle to candela
}

\author{
Yuqin Zong sheds light on photometry's fundamental unit.
}

T he candela (cd), the unit of luminous intensity, is the International System of Units (SI) base unit for photometry the science of measuring light as perceived by the human eye. The standards used for defining and measuring luminous intensity, a measure of the luminous power emitted by a light source, in a particular direction, per steradian, have evolved along with the development of technologies and application needs, from candles, gas lamps and incandescent lamps to Planck radiators. The candela was first defined in 1948, and reformulated in 1967 as "the luminous intensity, in the perpendicular direction, of a surface of $1 / 600,000$ square meter of a black body at the temperature of freezing platinum under a pressure of 101,325 newtons per square meter."

Subsequent advances in radiometry (the measurement of optical radiation power) brought new capabilities to the field of photometry, offering alternative ways to realize the candela in addition to using high-temperature Planck radiators, which are expensive and difficult to operate. Therefore, in 1979, the current definition of the candela was adopted": "The candela is the luminous intensity, in a given direction, of a source that emits monochromatic radiation of frequency $540 \times 10^{12}$ hertz and that has a radiant intensity in that direction of $1 / 683$ watt per steradian." The value $1 / 683 \mathrm{~W} \mathrm{sr}^{-1}$ was chosen to maintain continuity with the previous definitions based on candles and Planck radiators. Indeed, an ordinary wax candle produces nearly $1 \mathrm{~cd}$ of luminous intensity.

The 1979 definition is given for only one frequency of optical radiation. To determine the luminous intensity of a light source emitting monochromatic radiation with another frequency or a broad spectrum of frequencies, the International Commission on Illumination (CIE) has defined a set of weighting functions - called spectral luminous efficiency functions - that describe the relative spectral sensitivity of the average human eye for specified visual conditions (photopic, scotopic and mesopic vision). Using the definition of the candela and the CIE spectral luminous efficiency functions, the luminous intensity of any light source can be obtained.

In 2009, in response to advances in quantum-based optical technologies and their emerging metrological applications, the Consultative Committee for Photometry and Radiometry (CCPR) proposed to add the following explanatory text to the reformulation of the candela $a^{2}$ : "Thus we have the exact relation $K_{\mathrm{cd}}=683 \mathrm{~lm} / \mathrm{W}$. The effect of this definition is that the candela is the luminous intensity, in a given direction, of a source that emits monochromatic radiation of frequency $540 \times 10^{12}$ hertz and that has a radiant intensity in that direction of $1 / 683$ watt per steradian. This radiant intensity corresponds to a photon intensity of $(683 \times 540 \times$ $\left.10^{12} \times 6.62606896 \times 10^{-34}\right)^{-1}$ photons per second per steradian." (The constant $K_{\mathrm{cd}}$ involves the lumen $(\mathrm{lm}=\mathrm{cd} \mathrm{sr})$, the unit of luminous flux, a measure for the total amount of visible light emitted from a source. The unit 'photons per second per steradian' is used for photon intensity.) The last sentence of the explanatory text, which did not alter in any way the current definition of the candela, was a suggested compromise between proponents of a radiant-intensity formulation and those of a photon-intensity formulation ${ }^{3}$.

The radiant-intensity side's argument is that the current definition serves well the practical needs of the classical world of lighting and the majority of practitioners of photometry and radiometry ${ }^{3}$. Such a viewpoint implies, however, that direct realization of the candela using current photon-number-based techniques is difficult and has much larger uncertainties than the present method of using a cryogenic radiometer - an electrical-substitution radiometer that is operated at an extremely low temperature (typically, a few kelvin). The photon-intensity camp argues that proactive steps need to be taken to redefine the candela based on fundamental quanta (photons) to meet the increasing needs of quantum photometric and radiometric measurements. This view also expresses that human vision is photon-based - the interaction of photons with photoreceptor molecules in the eye.

In the end, there was no unanimous agreement within the CCPR concerning the 2009 proposal, and it was ultimately rejected. It is still unclear whether the ongoing debate on the explanatory text for the reformulation of the candela will eventually reach a consensus and whether a redefinition of the candela based on photons will be considered in the near future. Meanwhile, the CCPR decided to include photon-number-based quantities (for example, photon intensity, photon flux and photon irradiance) and their realizations in the recently published mise en pratique guidelines on how the candela and its related units can be realized in practice ${ }^{4}$.

In any event, $K_{\mathrm{cd}}$ is the new defining constant for photometry, without uncertainty, representing the luminous efficacy of monochromatic radiation of frequency $540 \times 10^{12}$ hertz, and is recommended by the CCPR to be one of the seven defining constants used to scale the entire SI system ${ }^{3,5}$.

A new SI brochure on the definitions of the SI units is now being prepared and is expected to be published in 2018, with a definition of the candela adjusted to the standard format for the definitions of all seven base units, that is, expressed in terms of its defining constant, $K_{\text {cd }}$.

YUQIN ZONG is at the National Institute of Standards and Technology, Gaithersburg, Maryland 20899, USA.

e-mail: yuqin.zong@nist.gov

\section{References}

1. SI Brochure: The International System of Units 8 th edn (BIPM, 2006).

2. CCPR Report of the 20th Meeting to the International

Committee for Weights and Measures (CIPM) (BIPM, 2009); http://go.nature.com/OXwCDR

3. Zwinkels, J. C. et al. Metrologia 47, R15-R32 (2010).

4. SI Brochure Appendix 2: Mise en Pratique for the Definition of the Candela and Associated Derived Units for Photometric and Radiometric Quantities in the International System of Units (SI) (BIPM, 2015)

5. Fischer, J. \& Ullrich, J. Nature Phys. 12, 4-7 (2016).

$\mathrm{m} e \AA \mathrm{s} \mathrm{u} R E_{\mathrm{h}}$

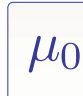

$\varepsilon_{0}$

$\alpha$

$\sigma$

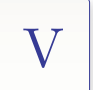

$\mathrm{R}$ 